\title{
Modelling of a CCP-RF Discharge Used For The Simulation Of Titan's Chemistry
}

\author{
L. Marques ${ }^{\mathrm{a}, \mathrm{b}}$, C.D. Pintassilgo ${ }^{\mathrm{a}, \mathrm{c}}$, G. Alcouffe ${ }^{\mathrm{d}}$, L.L. Alves ${ }^{\mathrm{a}}$, and \\ G.Cernogora ${ }^{\mathrm{d}}$ \\ "Instituto de Plasmas e Fusão Nuclear, Instituto Superior Técnico, 1049-001 Lisboa, Portugal \\ ${ }^{b}$ Centro de Física da Universidade do Minho, Universidade do Minho, 4710-057, Braga, Portugal \\ ${ }^{c}$ Depart. Eng. Física, Fac. Engenharia, Univ. Porto, R. Dr. Roberto Frias, 4200-465 Porto, Portugal \\ ${ }^{d}$ Université de Versailles St-Quentin Service d'Aéronomie, BP 3 - 91371 Verrières le Buisson France
}

\begin{abstract}
This paper reports the modelling of CCP-RF discharges $(13.56 \mathrm{MHz})$ in pure nitrogen, produced within a cylindrical parallel-plate reactor, similar to a GEC reference cell surrounded by a lateral grounded grid, at $0.1-2$ mbar pressures and $10-50 \mathrm{~W}$ coupled powers. This study is a first step in simulating Titan's chemistry at laboratory scale, using the PAMPRE experiment. Modelling results are compared with experimental measurements of the average electron density, and the self-bias potential at the polarized electrode.
\end{abstract}

Keywords: dusty plasma, light scattering, solid aerosols, Titan's atmosphere.

PACS: 52.27.LW; 42.25.Fx; 96.30nd

\section{INTRODUCTION}

In Titan's atmosphere, solid aerosols are produced by photochemistry reactions. Radio-Frequency Capacitively Coupled Plasma (CCP-RF) discharges in nitrogen/methane mixtures (for $\mathrm{CH}_{4}$ concentrations up to $10 \%$ ) can be used to simulate this chemistry, at laboratory scale. Actually, the CCP-RF device named PAMPRE [1] produces analogues of Titan's aerosols named "tholins", whose properties are presented in parent papers. Understanding the production of "tholins" is a very complex subject, to be carried out adopting a step-by-step methodology, whose starting point involves the analyses of plasmas with a simpler chemistry.

Here, we report the study of CCP-RF discharges $(13.56 \mathrm{MHz})$ in pure nitrogen, produced within a cylindrical parallel-plate reactor (with $69 \mathrm{~mm}$ radius and $45 \mathrm{~mm}$ inter-electrode distance), similar to a GEC reference cell surrounded by a lateral grounded grid, at 0.1-2 mbar pressures and 10-50 W coupled powers.

\section{MODEL DESCRIPTION}

We have used a two-dimensional $(r, z)$ time-dependent fluid-type code [2] to describe the transport of electrons and positive ions $\mathrm{N}_{2}{ }^{+}$and $\mathrm{N}_{4}{ }^{+}$in the reactor under study, coupled to a very complete zero-dimensional kinetic code for nitrogen gas [3]. 
The fluid code solves the charged particle continuity and momentum transfer equations, the electron mean energy transport equations, and Poisson's equation for the RF electric potential. The kinetic code solves the electron Boltzmann equation and the rate balance equations of 45 vibrationally excited states and 7 electronically excited states of the $\mathrm{N}_{2}$ molecule. Simulations yield the self-consistent DC-bias potential, the effective power coupled to the plasma, and the two-dimensional spatial distributions for (i) the densities and fluxes with the charged particles and the electron mean energy, (ii) the densities of the most relevant molecular states of nitrogen, and (iii) the RF plasma potential.

\section{RESULTS AND DISCUSSION}

First results reveal a strong coupling between discharge and kinetic features, as electrons are mainly produced via associative ionisation mechanisms involving the molecular metastable states $\mathrm{N}_{2}(A)$ and $\mathrm{N}_{2}$ (a'). Figures 1(a) and (b) plot the space-time average electron density and the self-bias potential, respectively, as a function of pressure at $30 \mathrm{~W}$ coupled power.
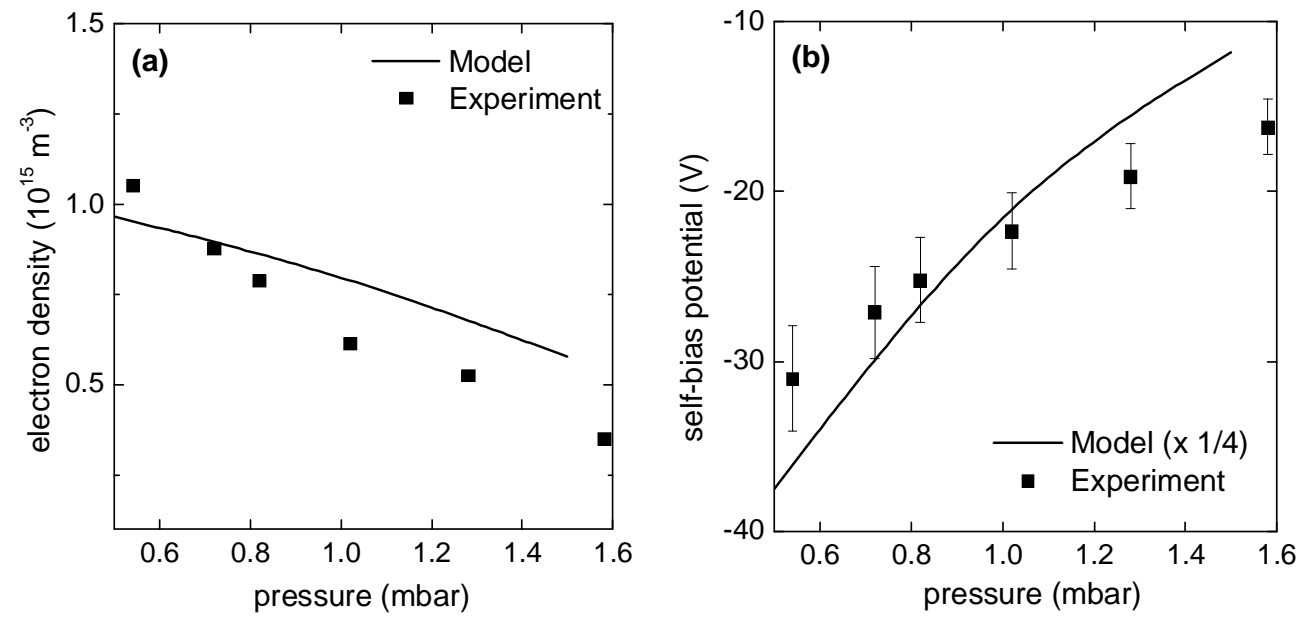

FIGURE 1. Space-time average electron density (a) and self-bias potential (b), as a function of pressure at $30 \mathrm{~W}$ coupled power.

As shown, there is a good agreement (both qualitative and quantitative) between simulations and resonant cavity measurements of the electron density [see Fig.1(a)]. However, calculations of the self-bias potential overestimate measurements by a factor of 4 [see Fig.1(b)], probably as a model response to an ion production overrate. Work is in progress to further analyse the effect of kinetics in model results, by extending the mechanisms accounting for the net production of charged particles.

\section{REFERENCES}

1. C. Szopa, G. Cernogora, L. Boufendi, J.-J. Correia and P. Coll, Planetary and Space Science 54, 394404 (2006).

2. A. Salabas, G. Gousset and L.L. Alves, Plasma Sources Sci. Technol. 11 448-465 (2002).

3. V. Guerra, P.A. Sá and J. Loureiro, Eur. Phys. J.: Appl. Phys. 28 125-152 (2004). 